\title{
On the role and value of $\beta$ in incompressible MHD simulations
}

\author{
Robert Chahine ${ }^{1}$ and Wouter J.T. Bos ${ }^{1}$ \\ 1 LMFA, CNRS, Ecole Centrale de Lyon - Université de Lyon - 69340 Ecully, \\ France
}

The parameter $\beta$, defined as the ratio of the pressure to the square of the magnetic field, is widely used to characterize astrophysical and fusion plasmas. However, in the dynamics of a plasma flow it is the pressure gradient which is important rather than the value of the pressure itself. It is shown that if one is interested in the influence of the pressure gradient on the dynamics of a plasma, it is not the quantity $\beta$ which should be considered, but a similar quantity, depending on the pressure gradient. The scaling of this newly defined quantity is investigated using incompressible magnetohydrodynamic simulations in a periodic cylinder in the Reversed Field Pinch flow regime.

\section{INTRODUCTION}

In conducting fluids and plasmas, the force-balance on a fluid element contains, in addition to the pressuregradient, also a magnetic pressure gradient. To measure their relative importance, one generally considers the ratio of the hydrodynamic to magnetic pressure, also called $\beta$. In astrophysical applications, the value of $\beta$ is used to characterize the plasma which is considered. Typically, low values of $\beta$ are observed in the solar wind, e.g. $\beta \ll 1$ in the mid-corona ${ }^{1}$. The value of $\beta$ in compressible magnetohydrodynamics (MHD) determines the precise behaviour and dimensionality of the system ${ }^{2}$. Furthermore, since $\beta$ is directly related to the magnetic Mach number, its value will determine the importance of magnetosonic compared to Alfvén wave propagation in a plasma ${ }^{3}$. In the remainder of this investigation we will focus on the use of $\beta$ in the magnetically confined fusion community, but the results and ideas are, we think, of broader relevance.

Let us first define $\beta$. Using Alfvénic units to normalize the plasma pressure and magnetic field (i.e. the plasma velocity is normalized by the Alfvén velocity $v_{A} \equiv B_{0} / \sqrt{\rho \mu_{0}}$, where $B_{0}$ is a characteristic value of the magnetic field, $\rho$ the plasma density and $\mu_{0}$ the magnetic permeability), $\beta$ is given by

$$
\beta=2 \frac{P}{B^{2}} .
$$

In magnetic fusion devices such as tokamaks or Reversed Field Pinches (RFPs), obtaining a high temperature and pressure in the center of the plasma is one of the indicators of good confinement. Indeed, the challenge is to confine a plasma as well as possible for a given magnetic field. The quantity $\beta$ gives then a rough measure of the reactor's efficiency, since it compares the pressure $P$ in the plasma, which indicates how high the temperature is, to the strength of the imposed magnetic field (characterized by $B^{2}$ ).

One particular definition of $\beta, \bar{\beta}$, is defined here as

$$
\bar{\beta}=2 \frac{\langle P\rangle}{\left\langle B^{2}\right\rangle},
$$

where the brackets indicate a volume average over the plasma volume of interest. Other definitions are also used $^{4}$ based on the value of $\boldsymbol{B}$ at the wall of the plasma for instance. The quantity $\beta$ is thus a key parameter in fusion design and operation, and further plays an important role in investigations of the stability of confined plasmas ${ }^{5}$ and the dynamics of magnetic islands in tokamaks ${ }^{6}$.

In many fusion related flows the value of $\beta$ is low, of the order of a few percent or even less, and as such, the effects of pressure are therefore sometimes neglected. This approximation was for instance used in investigations of the single-helicity states in the RFX reactor ${ }^{7,8}$, and in studies on helical modulation of the magnetic field at the boundary of the plasma ${ }^{9,10}$ and on sawtooth mitigation ${ }^{11}$. Also in the prediction of the relaxed Taylor-state ${ }^{12}$, the influence of $\beta$ was considered negligible. These are only few of the studies where the low- $\beta$ approximation is invoked to neglect the influence of pressure. This approximation, is in most of the more recent works not used, and it seems to be recognized by now that even when $\beta$ is small, the pressure cannot be neglected. Indeed, in most of the more recent numerical investigations of both tokamaks and RFPs the pressure is retained in the dynamics (see for instance in references ${ }^{13-17}$ reporting on sophisticated MHD codes for magnetically confined fusion applications).

The present study is not dedicated to the question whether finite $\beta$ effects are important or not, but how to efficiently quantify their importance. Hereby we contribute to the understanding of the counter-intuitive observation that, even for very small values of $\beta$, the pressure still is important in determining the precise dynamics. In other terms, we illustrate why the zero- $\beta$ (or constant pressure) description is not a good approximation of low- $\beta$ incompressible MHD simulations, no matter how small the value of $\beta$ is.

In the next section we will propose a new quantity, which we will call $\beta_{\nabla}^{\prime}$. In section III we will present numerical results which show that the new quantity is intrinsically different from the classical definition of $\beta$, since it has a radically different dependence on the Lundquist number. To further illustrate the importance of the new parameter, we show that finite $\beta$ simulations do not converge towards the zero- $\beta$ case for decreasing $\beta$. Indeed, decreasing $\beta$ does not necessarily imply decreasing $\beta_{\nabla}^{\prime}$. 


\section{IS $\beta$ A RELEVANT QUANTITY IN INCOMPRESSIBLE MHD SIMULATIONS?}

In the MHD description, the Navier-Stokes equations are modified by the presence of the Lorentz force $\boldsymbol{F}_{L}$. In Alfvénic units, this gives

$$
d_{t} \boldsymbol{u}=\nu \Delta \boldsymbol{u}-\nabla P+\boldsymbol{F}_{L} .
$$

where $\boldsymbol{u}(\boldsymbol{x}, t)$ is the velocity, $P(\boldsymbol{x}, t)$ the pressure and $\nu$ the viscosity. We consider for simplicity the incompressible isothermal formulation, where $\nabla \cdot \boldsymbol{u}=0$ and the density $\rho$ is uniform. The left hand side of this equation represents the acceleration of a plasma-fluid element. Through Newton's second law the right-hand-side gives the force-balance per unit mass. We see the contributions of three forces: viscous friction, the pressure gradient and the Lorentz-force. The Lorentz-force is given by

$$
\boldsymbol{F}_{L}=\boldsymbol{J} \times \boldsymbol{B},
$$

where the current density is given by the curl of the magnetic field $\boldsymbol{J}(\boldsymbol{x}, t)=\nabla \times \boldsymbol{B}(\boldsymbol{x}, t)$. Using vector identities, part of the Lorentz force can be written as the gradient of a scalar quantity, so that it can be regrouped with the pressure term. Doing so, equation (3) becomes

$$
d_{t} \boldsymbol{u}-\nu \Delta \boldsymbol{u}=-\frac{1}{2} \nabla\left(B^{2}(1+\beta)\right)+\boldsymbol{B} \cdot \nabla \boldsymbol{B},
$$

where $\beta$ is given by expression (1). It seems from this expression that if $P \ll B^{2}$, the hydrodynamic pressure drops out of the equation. This leads to the intuitive result that if in a plasma we measure a low value of $\beta$, the hydrodynamic pressure can be neglected. In this limit, (5) does not need the restriction $\nabla \cdot \boldsymbol{u}=0$ anymore and the description thereby simplifies.

We will here illustrate that this approximation is not straightforward. The important point is that in equation (5), it is the gradient of the pressure and of the magnetic pressure which play a role, and not the values of the pressures themselves. If one would like to measure the influence of the hydrodynamic pressure gradient compared to the gradient of the magnetic pressure, one needs to compare the norms of these vectors. Introducing the unit vectors

$$
\boldsymbol{e}_{P}=\frac{\nabla P}{\|\nabla P\|} \text { and } \boldsymbol{e}_{B}=\frac{\nabla B^{2}}{\left\|\nabla B^{2}\right\|}
$$

one can write equation (5) as

$$
d_{t} \boldsymbol{u}-\nu \Delta \boldsymbol{u}=-\frac{1}{2}\left\|\nabla B^{2}\right\|\left(\boldsymbol{e}_{B}+\boldsymbol{e}_{P} \beta_{\nabla}\right)+\boldsymbol{B} \cdot \nabla \boldsymbol{B} .
$$

In this expression $\beta_{\nabla}$ is defined by

$$
\beta_{\nabla} \equiv 2 \frac{\|\nabla P\|}{\left\|\nabla B^{2}\right\|} .
$$

The difference between expressions (5) and (7) is obviously the presence of the gradients in the definition of $\beta_{\nabla}$, which is essential in measuring the effects of pressure on the dynamics. Evidently a large uniform pressure will not induce any acceleration of the plasma and one can thus always change the value of the pressure without changing its gradient by adding a uniform pressure field.

Furthermore, the separation of the Lorentz-force into a gradient $\nabla\left(B^{2} / 2\right)$ and $\boldsymbol{B} \cdot \nabla \boldsymbol{B}$ seems natural when we want to consider the influence of the pressure, and it is so in the absence of motion, when the left-hand-side of equation (3) is zero. Computing subsequently an equilibrium field by considering a force-free magnetic state is a classical way to determine tokamak equilibria, already widely used in the early days of fusion research ${ }^{18,19}$, and plasma physics in general ${ }^{20}$. For this static case we have thus

$$
\frac{1}{2} \nabla\left(B^{2}(1+\beta)\right)=\boldsymbol{B} \cdot \nabla \boldsymbol{B},
$$

and for small values of $\beta$, only the magnetic pressure can balance $\boldsymbol{B} \cdot \nabla \boldsymbol{B}$. However, if one focuses on the importance of the pressure on the nonlinear dynamics in equation (7), i.e. when $\boldsymbol{u} \neq 0$, there is no reason, other than esthetics, to separate the Lorentz-force in two parts. Indeed, as soon as the plasma will start to move, the acceleration $d \boldsymbol{u} / d t$ of the plasma will be governed by the pressure gradient, the Lorentz-force and the viscous stress, and the pressure gradient is no longer a priori negligible.

Note that we can ignore the contribution of the viscous diffusion of momentum on the acceleration when the dynamics become turbulent ${ }^{21,22}$. This is plausible to hold in the present case for large values of the Lundquist number. Indeed, viscous dissipation is an essential feature in the eventual dissipation of kinetic energy of turbulent flows, but its influence on the acceleration is small, when eddy diffusion becomes more important than molecular viscous diffusion. We will not further consider it here.

What we will consider is the comparison of the forces due to pressure, and the Lorentz-force. Therefore we rewrite the momentum equation as,

$$
d_{t} \boldsymbol{u}-\nu \Delta \boldsymbol{u}=\left(-\boldsymbol{e}_{P} \beta_{\nabla}^{\prime}+\boldsymbol{e}_{F}\right)\|\boldsymbol{J} \times \boldsymbol{B}\|,
$$

where $\boldsymbol{e}_{F}=\boldsymbol{F}_{L} /\left\|\boldsymbol{F}_{L}\right\|$, and

$$
\beta_{\nabla}^{\prime} \equiv \frac{\|\nabla P\|}{\|\boldsymbol{J} \times \boldsymbol{B}\|} .
$$

In order to have global measures of the importance of the different terms, we introduce, as in expression (2), the volume averaged quantities,

$$
\bar{\beta}_{\nabla} \equiv 2 \frac{\langle\|\nabla P\|\rangle}{\left\langle\left\|\nabla B^{2}\right\|\right\rangle} \text { and } \overline{\beta_{\nabla}^{\prime}} \equiv \frac{\langle\|\nabla P\|\rangle}{\langle\|\boldsymbol{J} \times \boldsymbol{B}\|\rangle} .
$$

It is only when $\overline{\beta_{\nabla}^{\prime}}$ is small that the pressure term might be negligible in the dynamics. However, if this quantity is of order unity, there is no reasonable indication that the pressure gradient can be safely ignored. We will now assess this in the context of RFP simulations and we will show that $\beta$ and $\beta_{\nabla}^{\prime}$ behave differently. 


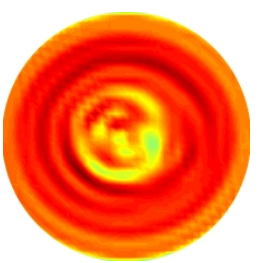

(a)

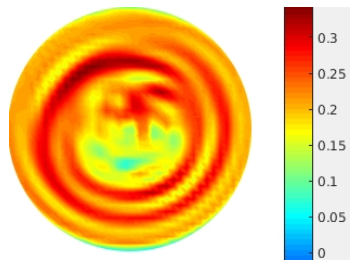

(b)
FIG. 1. Visualization of (a) $z=0$ (b) $z=L / 2$ cross-sections of the pressure field in the RFP in the turbulent state $S=$ 33600 and $\Theta=7$, in the statistically steady state.

\section{RESULTS FOR $\beta$ AND $\beta_{\nabla}$ IN STRAIGHT-CYLINDER RFP SIMULATIONS}

\section{A. Numerical set-up}

In this section we will evaluate how $\bar{\beta}, \overline{\beta_{\nabla}}$ and $\overline{\beta_{\nabla}^{\prime}}$ scale in incompressible MHD simulations in a periodic cylinder with aspect ratio $\Gamma=L_{z} / 2 \pi r=4$, where $L_{z}$ and $r=1$ are the cylinder length and radius, respectively. Equation (7) is solved together with the induction equation,

$$
\frac{\partial \boldsymbol{B}}{\partial t}=\nabla \times(\boldsymbol{u} \times \boldsymbol{B})+\lambda \nabla^{2} \boldsymbol{B}
$$

and the condition $\nabla \cdot \boldsymbol{B}=0$. Computations are performed using a pseudo-spectral solver on a Cartesian mesh of $512 \times 128 \times 128$ gridpoints, combined with a volume-penalization technique to enforce the boundary conditions. The boundary conditions are no-slip on the cylinder wall for the velocity. The magnetic field is enforced by imposing the poloidal component of the magnetic field $B_{p}$ at the wall, which thereby determines the global axial current. The radial magnetic field vanishes at the wall and the axial component $B_{z}$ consists of a uniform, fixed contribution plus a variable part which is left free to evolve, so that the magnetic field can self-organize towards a typical RFP dynamic equilibrium. Details on the numerical method can be found $\mathrm{in}^{23}$.

The pressure field is calculated using the incompressibility condition by solving the Poisson equation obtained from taking divergence of the momentum equation, expression (3),

$$
\nabla^{2} P=\nabla \cdot(-\boldsymbol{u} \cdot \nabla \boldsymbol{u}+\boldsymbol{J} \times \boldsymbol{B})
$$

where the time-derivative and viscous term vanish due to incompressibility. In a pseudo-spectral code, this Poisson equation can be easily solved using Fourier transforms. The boundary condition for this resolution is zero pressure on the plasma boundaries. In all simulations, we will consider the RFP regime where the magnetic geometry is self-organized into a helical structure. Typical similations using the same code and set-up are reported $i^{24}$. In Figure 1 we show visualizations of the pressure field in cross-sections of the cylinder for a typical flow.

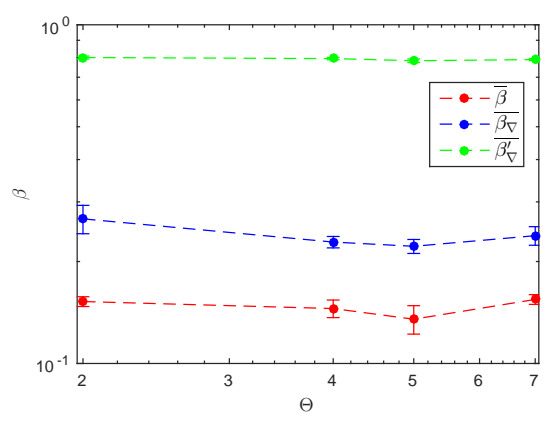

FIG. 2. Importance of the pressure force on the dynamics as measured by $\bar{\beta}, \overline{\beta_{\nabla}}$ and $\overline{\beta_{\nabla}^{\prime}}$ for different values of the pinch ratio with $S=2.8 \times 10^{4}$.

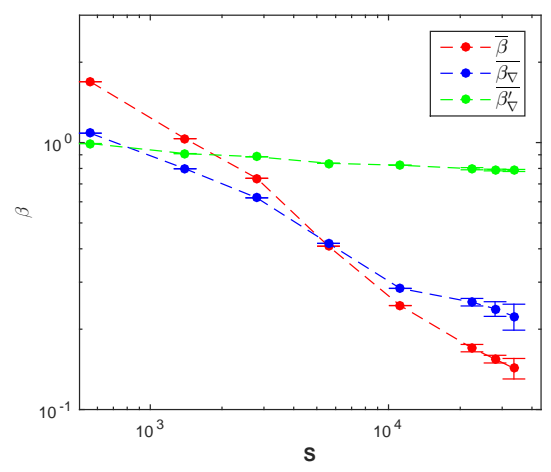

FIG. 3. Importance of the pressure force on the dynamics as measured by $\bar{\beta}, \overline{\beta_{\nabla}}$ and $\overline{\beta_{\nabla}^{\prime}}$ for different values of the Lundquist number.

\section{B. Influence of the pinch-ratio}

We consider first the case of a fixed Lundquist number, $S \equiv B_{p} r / \lambda=2.8 \times 10^{4}, P_{m} \equiv \nu / \lambda=1$ for different values of the pinch $\Theta=\bar{B}_{p} /\left\langle B_{z}\right\rangle$, where the overline indicates the surface average on the edge of the plasma and the brackets indicate a volume average. Error-bars here and in the following measure the temporal fluctuations of the evaluated quantities during the statistically stationary state, during which the quantities are evaluated.

In figure 2 the evolution of the different definitions of $\beta$ is shown to stay constant with respect to the pinch ratio. Furthermore, $\bar{\beta}$ takes a value less than $20 \%$ while the quantity $\overline{\beta_{\nabla}^{\prime}}$ is greater than $80 \%$. For the parameters considered here, the value of $\bar{\beta}, \overline{\beta_{\nabla}}$ and $\overline{\beta_{\nabla}^{\prime}}$ all three seem to be roughly independent of the pinch-ratio. Considering only this result, one might be tempted to conclude that all three quantities play a similar role, so that low $\beta$ dynamics are equivalent to low $\overline{\beta_{\nabla}}$ dynamics. That this is not so will now be shown. 


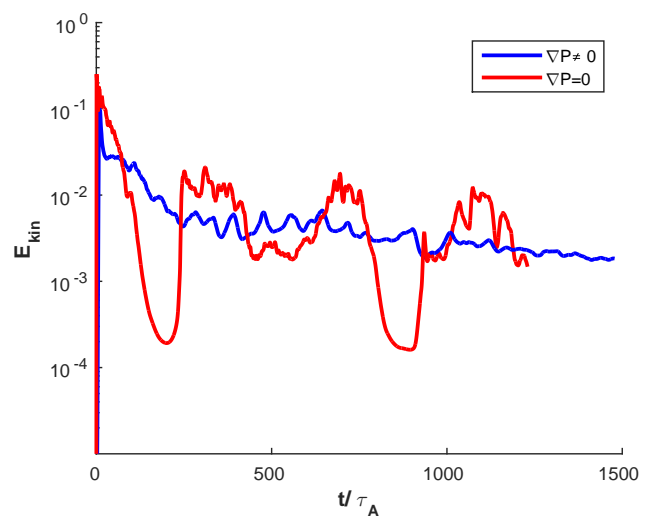

FIG. 4. Time evolution of the kinetic energy for simulations with $S=22400 \Theta=7$, in the presence (blue) and absence (red) of the pressure gradient.

\section{Influence of the Lundquist number}

We now turn to the dependence of the different $\beta \mathrm{s}$ on the Lundquist number. In Figure 3 it is observed that increasing $S, \bar{\beta}$ drops, approximately following a powerlaw proportional to $S^{-1 / 2}$. Judging from this dependence only and extrapolating, one would be tempted to say that in realistic regimes, where $S$ is at least an order of magnitude higher, the pressure is negligible. However, considering the influence of the pressure gradient on the force balance by comparing the pressure-gradient to the Lorentz-force, it is observed that the influence of the pressure force remains comparable to the Lorentzforce for all values of $S$. Indeed $\overline{\beta_{\nabla}^{\prime}}$ remains order unity when $S$ is increased, while $\overline{\beta_{\nabla}}$ remains between the two other quantities.

The pressure gradient is thus not negligible in the dynamics, and this is the main result of the present work: observation of a low value of $\beta$ does not mean that the pressure gradient can be neglected in the dynamics. A question is now how the results change if the we neglect the pressure anyway. This will be illustrated in the following.

\section{ON THE DIFFERENCE BETWEEN INCOMPRESSIBLE AND ZERO- $\beta$ SIMULATIONS}

To illustrate the importance of the foregoing we will show here that finite $\beta$ simulations are radically different from zero- $\beta$ simulations. Indeed, this is implicitly accepted in the fusion community and the pressure is retained in most of the recent investigations of tokamak and RFP dynamics even when $\beta$ is small. However the novelty here is that this difference can be directly measured using the quantity $\overline{\beta_{\nabla}^{\prime}}$.

In order to illustrate this we compare the foregoing results with results obtained in the absence of pressure.

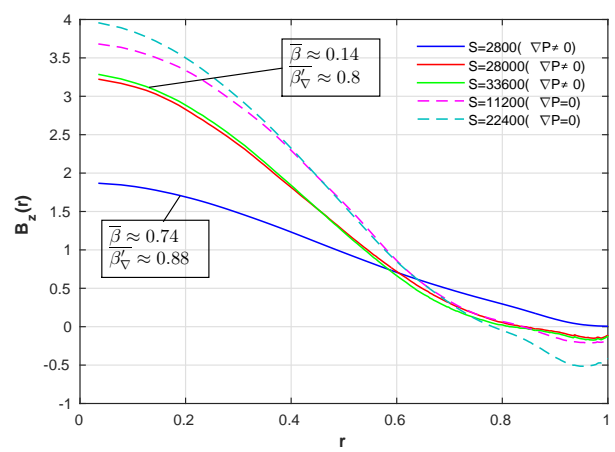

FIG. 5. Importance of the pressure force to determine the magnetic field for different values of the Lundquist number.

The system we consider is thus

$$
d_{t} \boldsymbol{u}-\nu \Delta \boldsymbol{u}=\boldsymbol{J} \times \boldsymbol{B},
$$

combined with equation (13). A similar comparison was reported in ${ }^{25}$. A theoretical study ${ }^{26}$ explained some of the differences between the results. More recently zero- $\beta$ and finite- $\beta$ simulations were compared in the context of $\mathrm{RFP}$ relaxation in reference ${ }^{27}$.

In Figure 4 we show the evolution of the kinetic energy $\left(E_{\text {kin }}=\left(|\boldsymbol{u}|^{2}\right) / 2\right)$ for two simulations at $S=22400$, $\Theta=7$. It is observed that the dynamics are more violently fluctuating in the pressureless simulations. This is somehow expected, since pressure is known to redistribute momentum over different velocity components, thereby smoothing the flow with respect to pressureless dynamics. In the absence of a magnetic field this is a well-known difference between Navier-Stokes dynamics and the dynamics of Burgers' equation, associated with the generation of shocks. Indeed, the behavior of the pressureless equations should be very similar to the limit of very high compressibility ${ }^{28}$.

In Figure 5 we show the average axial magnetic field as a function of the radial coordinate. We compare the results from incompressible and zero- $\beta$ simulations for Lundquist numbers ranging from $S=11200$ upto $S=33600$. The main observation is that we have a dichotomy where the profiles of the simulations without pressure are qualitatively different from the full incompressible dynamics. For both cases the profiles become roughly independent of $S$ for $S>10^{4}$, but the asymptotic profiles are different with a deeper reversal for the pressureless dynamics.

For the incompressible runs we have a $\beta$ which decreases as a function of $S$, as shown in Figure 3. We observe that between the lowest and the highest values of the Lundquist number, $\beta$ has decreased by an order of magnitude. However, even at the highest value of $S$, the radial profile of the axial magnetic field has not approached the incompressible solution towards the zero- $\beta$ solution. This clearly illustrates that the pressure plays an important role and that it is not $\beta$ which can measure 
the importance of the pressure force on the dynamics. Indeed the value of $\overline{\beta_{\nabla}^{\prime}}$ is of order unity for all the incompressible simulations. Therefore, considering $\overline{\beta_{\nabla}^{\prime}}$ one would be less tempted to ignore the pressure term, since it remains of order unity, even when $\bar{\beta}$ decreases.

\section{v. CONCLUSION}

We have argued that the quantity which measures the importance of the pressure on the dynamics in incompressible MHD simulations is $\overline{\beta_{\nabla}^{\prime}}=\langle\|\nabla P\|\rangle /\langle\|\boldsymbol{J} \times \boldsymbol{B}\|\rangle$. Indeed, it is shown that even when $\beta$ might vary considerably, in particular when increasing the Lundquist number, the value of $\overline{\beta_{\nabla}^{\prime}}$ remains of order unity and the influence of the pressure gradient cannot be neglected. Our results considered the simplest case of incompressible isothermal flow. We have not carried out simulations of compressible MHD, but we expect that the coupling between the $\beta$ quantities and the Lundquist number will be affected and that their dependence wil be somewhat weaker. Furthermore, we expect that the general behaviour in compressible similations will qualitatively be intermediate between those of the pressureless and the incompressible cases considered in the previous section. Assessing this by complexifying the description is however an interesting issue to be further explored.

These results do not completely invalidate the insights obtained in the zero- $\beta$ simulations. Indeed, a lot of qualitative features observed without pressure can carry over to the actual incompressible MHD dynamics. Some of this coincidence is fortunate and it would be wise to validate these results using more a more complete MHD description involving the pressure term. However, MHD is already a very simplified description of the actual plasma and ignoring the pressure gradient is only one approximation among many others (for instance, non-uniform resistivity influences the RFP dynamics importantly ${ }^{29,30}$ ). It is our viewpoint that MHD simulations should be considered as tools to discover possible physical mechanisms, which can be assessed in realistic experiments. Furthermore, neglecting the pressure in MHD is less dramatic than in hydrodynamics. Indeed, the presence of the magnetic pressure will take over part of the role normally played by the hydrodynamic pressure. This is not so in the absence of a magnetic field, where the pressureless equations (Burger's equation) will lead to a far more extreme dynamics with large gradients and shocks which change completely the flow-dynamics. However even in the present case the pressureless dynamics are more violent than the full incompressible system, as illustrated in Figure 4.

We conclude by stressing that, by no means, we claim that $\beta$ is an unimportant parameter to characterize a plasma, neither do we criticise the zero- $\beta$ assumption as a simplifying hypothesis. What should be retained from this study is that the importance of the pressure forces can directly be measured by comparing the pressure gra- dient term to the Lorentz-force. The resulting quantity $\overline{\beta_{\nabla}^{\prime}}$ which measures this is intrinsically different from $\bar{\beta}$, which is for instance illustrated by a markedly different dependence on the Lundquist number.

\section{ACKNOWLEDGMENTS}

Simulations were carried out using the facilities of the PMCS2I (École Centrale de Lyon), IDRIS (Project No. 22206) and P2CHPD. Support of the French Research Federation for Fusion Studies (FR-FCM) is acknowledged.

${ }^{1} \mathrm{G}$ Allen Gary. Plasma beta above a solar active region: rethinking the paradigm. Solar Physics, 203(1):71-86, 2001.

${ }^{2}$ Go P Zank and WH Matthaeus. Nearly incompressible fluids. ii: Magnetohydrodynamics, turbulence, and waves. Physics of Fluids A: Fluid Dynamics, 5(1):257-273, 1993.

3 Nahuel Andrés, Patricio Clark di Leoni, Pablo D Mininni, Pablo Dmitruk, Fouad Sahraoui, and William H Matthaeus. Interplay between alfvén and magnetosonic waves in compressible magnetohydrodynamics turbulence. Physics of Plasmas, 24(10):102314, 2017.

${ }^{4} \mathrm{JA}$ Wesson. Hydromagnetic stability of tokamaks. Nuclear Fusion, 18(1):87, 1978.

${ }^{5} \overline{\mathrm{R} \text {. Paccagnella, A. Bondeson, and H. Lütjens. Ideal toroidal }}$ stability beta limits and shaping effect for reversed field pinch configurations. Nucl. Fusion, 31(10), (1991).

${ }^{6}$ Magali Muraglia, Olivier Agullo, Masatoshi Yagi, Sadruddin Benkadda, P Beyer, Xavier Garbet, S-I Itoh, Kimitaka Itoh, and Abhijit Sen. Effect of the curvature and the $\beta$ parameter on the nonlinear dynamics of a drift tearing magnetic island. Nuclear Fusion, 49(5):055016, 2009.

${ }^{7}$ S. Cappello and D. F. Escande. Bifurcation in viscoresistive MHD: The hartmann number and the reversed field pinch. Phys. Rev. Lett., 85, (2000).

${ }^{8} \overline{\mathrm{DF}}$ Escande, R Paccagnella, S Cappello, C Marchetto, and F D'Angelo. Chaos healing by separatrix disappearance and quasisingle helicity states of the reversed field pinch. Phys. Rev. Lett., 85, (2000).

${ }^{9} \overline{\mathrm{D}}$ Bonfiglio, M Veranda, S Cappello, DF Escande, and L Chacón. Experimental-like helical self-organization in reversed-field pinch modeling. Physical review letters, 111(8):085002, 2013.

${ }^{10} \mathrm{M}$ Veranda, D Bonfiglio, S Cappello, L Chacón, and DF Escande. Impact of helical boundary conditions on nonlinear $3 \mathrm{~d}$ magnetohydrodynamic simulations of reversed-field pinch. Plasma Physics and Controlled Fusion, 55(7):074015, 2013.

${ }^{11}$ D Bonfiglio, M Veranda, S Cappello, L Chacón, and DF Escande. Sawtooth mitigation in $3 \mathrm{~d}$ mhd tokamak modelling with applied magnetic perturbations. Plasma Physics and Controlled Fusion, 59(1):014032, 2016.

${ }^{12} \mathrm{~J}$. B. Taylor. Relaxation and magnetic reconnection in plasmas. Rev. Mod. Phys., 58, (1986).

13 AH Glasser, CR Sovinec, RA Nebel, TA Gianakon, SJ Plimpton, MS Chu, DD Schnack, and NIMROD Team. The nimrod code: a new approach to numerical plasma physics. Plasma Physics and Controlled Fusion, 41(3A):A747, 1999.

${ }_{14}$ Stephen C Jardin, J Breslau, and N Ferraro. A high-order implicit finite element method for integrating the two-fluid magnetohydrodynamic equations in two dimensions. Journal of Computational Physics, 226(2):2146-2174, 2007.

${ }_{15}$ GTA Huysmans and O Czarny. Mhd stability in x-point geometry: simulation of elms. Nuclear fusion, 47(7):659, 2007.

${ }^{16}$ Daniele Bonfiglio, Luis Chacón, and Susanna Cappello. Nonlinear three-dimensional verification of the specyl and pixie3d magnetohydrodynamics codes for fusion plasmas. Physics of Plasmas, 17(8):082501, 2010. 
${ }^{17}$ Hinrich Lütjens and Jean-François Luciani. Xtor-2f: a fully implicit newton-krylov solver applied to nonlinear $3 \mathrm{~d}$ extended mhd in tokamaks. Journal of Computational Physics, 229(21):81308143, 2010.

${ }^{18}$ Harold Grad and Hanan Rubin. Hydromagnetic equilibria and force-free fields. Journal of Nuclear Energy (1954), 7(3-4):284285, 1958.

${ }^{19}$ VD Shafranov. Plasma equilibrium in a magnetic field. Reviews of Plasma Physics, 2:103, 1966.

${ }^{20}$ Lodewijk Woltjer. A theorem on force-free magnetic fields. Proceedings of the National Academy of Sciences, 44(6):489-491, 1958

${ }^{21}$ Arkady Tsinober, Prakash Vedula, and PK Yeung. Random taylor hypothesis and the behavior of local and convective accelerations in isotropic turbulence. Physics of Fluids, 13(7):1974-1984, 2001.

${ }^{22}$ Mark Nelkin. Universality and scaling in fully developed turbulence. Advances in physics, 43(2):143-181, 1994.

${ }^{23}$ J. A. Morales, M. Leroy, W. J.T. Bos, and K. Schneider. Simulation of confined magnetohydrodynamic flows with Dirichlet boundary conditions using a pseudo-spectral method with volume penalization. J. Comp. Phys., 274, (2014).
${ }^{24}$ J. A. Morales, W. J.T. Bos, K. Schneider, and D. Montgomery. On the effect of toroidicity on reversed field pinch dynamics. Plasma Phys. Control. Fusion, 56, (2014).

${ }_{25}$ AY Aydemir, DC Barnes, EJ Caramana, AA Mirin, RA Nebel, DD Schnack, and AG Sgro. Compressibility as a feature of field reversal maintenance in the reversed-field pinch. The Physics of fluids, 28(3):898-902, 1985.

${ }^{26}$ John M Finn, Rick Nebel, and Charles Bathke. Single and multiple helicity ohmic states in reversed-field pinches. Physics of Fluids B: Plasma Physics, 4, (1992).

27 JR King, CR Sovinec, and VV Mirnov. First-order finite-larmorradius fluid modeling of tearing and relaxation in a plasma pinch a. Physics of Plasmas, 19(5):055905, 2012.

${ }^{28}$ Tomomasa Tatsumi and Hiroshi Tokunaga. One-dimensional shock turbulence in a compressible fluid. Journal of Fluid Mechanics, 65(3):581-601, 1974.

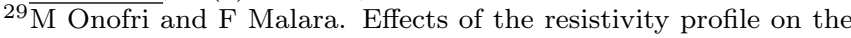
formation of a reversed configuration and single helicity states in compressible simulations of the reversed-field pinch. Physics of Plasmas, 20(10):102514, 2013.

${ }^{30}$ S. Futatani, J. A. Morales, and W. J.T. Bos. Dynamic equilibria and magnetohydrodynamic instabilities in toroidal plasmas with non-uniform transport coefficients. Phys. Plasmas, 22, (2015). 